\title{
CONVECTIVE HEAT TRANSFER ON STENOSED BLOOD FLOW THROUGH PERMEABLE MICROCIRCULATION IN THE PRESENCE OF A MAGNETIC FIELD
}

Alana Sankar $^{1 *}$, Sreedhara Rao Gunakala ${ }^{2}$ and Donna M. G. Comissiong ${ }^{3}$

1,2,3 Faculty of Science and Technology, The University of the West Indies, Trinidad

${ }^{1}$ Email: alana.sankar@sta.uwi.edu*(Corresponding author)

${ }^{2}$ Email: sreedhara.rao@sta.uwi.edu

${ }^{3}$ Email: donna.comissiong@sta.uwi.edu

\begin{abstract}
Blood flow through permeable microcirculation in the presence of a composite stenosis, an external magnetic field and convective heat transfer was examined. A twolayered model for the blood consisting of a fluid-particle suspension in the core region with a peripheral cell-free plasma layer was used. The proposed system of equations was solved and plots were generated. In the presence of permeable walls, an external magnetic field and convective heat transfer, the temperature of the blood, friction-factor Reynolds number and Nusselt number were investigated. The temperature of the blood increased when the Hartmann number increased, Darcy number increased, haematocrit level increased or the peripheral layer thinned. The friction-factor Reynolds number product increased as the haematocrit, Hartmann number, stenosis height or Darcy number increased. The Nusselt number decreased as the Hartmann number, haematocrit, stenosis height or Darcy number increased. These results were interpreted in terms of the physical situation. This study aids in understanding the effects of wall permeability, a magnetic field and the presence of heat transfer on different diseased arterial systems in the future.
\end{abstract}

Keywords: Blood flow, Heat transfer, Magnetic field, Permeable microcirculation.

\section{https://doi.org/10.47412/VYBY3868}

\section{Nomenclature}

$\begin{array}{llll}z & \text { Axis along the tube } & p & \text { Pressure } \\ r & \text { Axis normal to the tube axis } & a_{0} & \text { Erythrocyte radius } \\ R_{l} & \text { Radius of central layer } & u_{B} & \text { slip velocity } \\ \left(R-R_{l}\right) & \text { Radius of surrounding Newtonian fluid layer } & \mu_{s} & \text { Suspension viscosity } \\ \delta & \text { Maximum height of stenosis } & M & \text { Hartmann number } \\ \delta_{1} . & \text { Maximum height of bulge at the interface } & D a & \text { Darcy number } \\ \alpha & \text { Ratio of radius of central core to the tube } & P_{c} & \text { Peclet number } \\ C & \text { Volume fraction density of the particles } & \alpha_{s} & \text { slip parameter } \\ (p, f) & \text { Quantities related to (particle, fluid) phases } & K & \text { medium's permeability } \\ \sigma & \text { Electrical conductivity of the fluid } & \zeta & \text { ratio of diffusivities } \\ B_{0} & \text { Component of the magnetic field applied } & \varphi & \text { ratio of conductivities } \\ T & \text { Blood's temperature (in Kelvin) } & k_{0} & \text { plasma thermal conductivity } \\ S & \text { Modified drag coefficient of interaction } & T_{i} & \text { temperature in the inner region } \\ k_{f} & \text { thermal conductivity of fluid } & T_{0} & \text { temperature in the outer region } \\ N u & \text { average Nusselt number } & T_{m} & \text { mean temperature }\end{array}$


$\left(u_{f}, u_{p}, u_{0}\right) \quad$ Velocity axial components of (core fluid, particle, peripheral fluid)

\section{Introduction}

Studies involving an examination of different effects on blood flow through a blood vessel with stenosis are crucial in understanding what happens as blood flows in the vessels of patients with atherosclerosis. Since cells and organs are extremely sensitive to temperature changes, it is important to identify factors which can alter the temperature of blood. Temperature distribution during hyperthermia in large blood vessels has been presented by Lagendijk [1] and was in keeping with the experimental results of Torell and Nilsson [2]. From this, many theoretical studies involving heat transfer on blood have followed. Forced convection heat transfer was introduced to a laminar flow in various parallel porous channels to examine the effect of the porous channel [3]. Since vessel walls in microcirculation are permeable, this feature must be included in such studies.

A similar numerical study of the thermal performance in a microchannel was conducted by Raisi, Ghasemi and Aminossadati [4] which included both slip and no-slip conditions. Temperature jump was not considered as the flow in a microchannel was being examined. It was revealed that heat transfer rate was affected by varying the solid volume fraction and slip velocity coefficient at high Reynolds numbers. Viscous dissipation effects have been included when examining slip magnetohydrodynamic (MHD) flow and heat transfer past a permeable surface [5]. Hydromagnetic and thermal slip flow boundary layers over a flat plate was studied by Rahman [6] which included convective boundary conditions. Temperature jump conditions due to slip flow were neglected which was reasonable following the observations of Martin and Boyd [7].

The Bio-heat equation can also be used in the study of heat transfer in blood [8]. Heat from a heat source has also been included on a MHD blood flow [9]. The micropolar fluid was used by Das [10] to examine slip effects on MHD flow toward a shrinking vertical sheet and then by Mahmoud and Waheed [11] for MHD and heat transfer with heat generation and slip velocity over a stretching surface. Using the Bingham Non-Newtonian fluid, MHD peristaltic flow in an eccentric annulus was researched and temperature was found to increase with increasing Hartmann number [12]. Two phase flow in the presence of stenosis and heat transfer concluded that heat increased with the haematocrit parameter as well as with viscous heating [13].

Other studies involving MHD and heat transfer used the nanofluid. MHD boundary layer flow with heat transfer was considered over a convectively heated non-linear permeable sheet by Daniel [14]. Radiative heat transfer on MHD blood flow in an inclined tapered stenosed porous artery was examined [15].

Blood has been modelled as a nanofluid through an artery with composite stenosis and permeable walls in the presence of heat transfer only [16]. Tripathi and Kumar Sharma [17] explored MHD flow of blood through an inclined porous artery with heat transfer using a Newtonian fluid model. However due to the nature of blood, a two-layered model is suitable for examining flow in microcirculation [18, 19]. The effect of heat transfer on two-layered blood flow through a composite stenosis in the presence of a magnetic field has been researched by Sankar, Rao Gunakala and Comissiong [20] but the permeability of the vessel wall was not considered. Thus, it is critical that blood flow through stenosis with permeable microcirculation be considered when examining blood flow in the presence of an external magnetic field and heat transfer.

In this study, the effects of wall permeability in the presence of an external magnetic field and forced convective heat transfer were examined. A two-layered flow through a composite stenosis with permeable vessel walls was utilized. Temperature profiles were generated and the effect of wall permeability, the magnetic field, haematocrit and stenosis height on the friction-factor Reynolds number and Nusselt number were examined in the presence of the stenosis. 


\section{Methodology}

Consider the two-layered model for blood flow in microcirculation in the presence of a mild, axially nonsymmetric and radially symmetric composite stenosis, permeable walls and an external magnetic field. The blood vessel is assumed to be circular, uniform and of semi-infinite extent with rigid walls. The wall near where the stenosis if formed was assumed to be solid. Entrance, end and special wall effects are not considered since it is also assumed that the length of the vessel is much greater than its radius. Blood was represented by a two-layered model consisting of a central layer of erythrocytes suspended in plasma and a surrounding Newtonian fluid layer. To this however, heat transfer in the form of forced convection occurs.

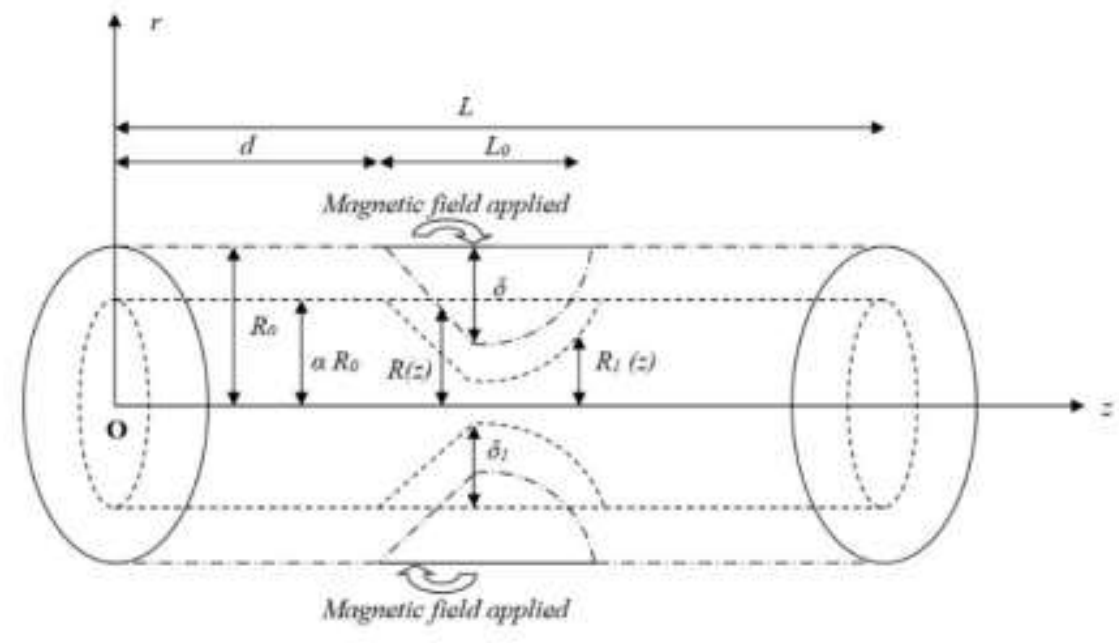

Figure 1: The geometry of the problem.

After non-dimensionalising and assuming further that $\left(\frac{\delta}{R_{0}}\right) \ll 1, \operatorname{Re}\left(\frac{\delta}{L_{0}}\right) \ll 1$ and $\left(\frac{R_{0}}{L_{0}}\right) \cong O(1)$ for a mild stenosis, the following equations were derived. The geometry of the stenosis manifested [20] in Fig. 1 is

$$
\left(R, R_{1}\right)=\left\{\begin{array}{c}
(1, \alpha)-2\left(\delta, \delta_{1}\right)(z-d) ; d \leq z \leq d+\frac{1}{2} \\
(1, \alpha)-\frac{\left(\delta, \delta_{1}\right)}{2}\left\{1+\cos 2 \pi\left(z-d-\frac{1}{2}\right)\right\} ; d+\frac{1}{2} \leq z \leq d+1 \\
(1, \alpha) ; \text { otherwise }
\end{array}\right.
$$

The stenosis starts at $z=d$ and reaches its maximum height where $\mathrm{z}=\mathrm{d}+\frac{1}{2}$. Including the presence of the Lorentz's force, in the core region, $\left(0 \leq r \leq R_{l}\right)$, a macroscopic two-phase model was used and in the peripheral region, $R_{l} \leq r \leq R$, a Newtonian fluid model.

The equations governing the blood flow are given by

$$
\begin{gathered}
(1-C) \frac{d p}{d z}=(1-C) \frac{\mu_{s}}{r} \frac{\partial}{\partial r}\left(r \frac{\partial u_{f}}{\partial r}\right)+C S\left(u_{p}-u_{f}\right)-M^{2} u_{f}, R_{1} \leq r \leq R \\
C \frac{d p}{d z}=C S\left(u_{f}-u_{p}\right), R_{1} \leq r \leq R \\
\frac{d p}{d z}=\frac{1}{r} \frac{\partial}{\partial r}\left(r \frac{\partial u_{0}}{\partial r}\right)-M^{2} u_{0}, 0 \leq r \leq R_{1}
\end{gathered}
$$


where $M=\left(\sqrt{\frac{\sigma B_{0}^{2} R_{0}^{2}}{\mu_{0}}}\right)$ is the Hartmann number. The suspension viscosity is given by $\mu_{s}(C)=\frac{1}{1-m C}$, where $m=\left(7 \times 10^{-2}\right) \exp \left[2.49 C+\left(\frac{1107}{T}\right) \exp (-1.69 C)\right][16]$.

The modified drag coefficient of interaction is $S=4.5\left(\frac{1}{a_{0}^{2}}\right) \frac{4+3\left(8 C-3 C^{2}\right)^{\frac{1}{2}}+3 C}{(2-3 C)^{2}}[17]$.

In this case, the boundary conditions are given as

$$
\begin{gathered}
\frac{\partial u_{f}}{\partial r}=\frac{\partial u_{p}}{\partial r}=0, \text { at } r=0, \\
u_{0}=u_{f}, \text { at } r=R_{1}, \\
\frac{\partial u_{0}}{\partial r}=(1-C) \mu_{s} \frac{\partial u_{f}}{\partial r}, \text { at } r=R_{1}, \\
u_{0}=u_{B}, \text { at } r=R, \\
\frac{\partial u_{0}}{\partial r}=\frac{\alpha_{s}}{\sqrt{D_{a}}}\left(u_{B}+D_{a}\right) \frac{d p}{d z}, \text { at } r=R_{1},
\end{gathered}
$$

where $D_{a}\left(=\frac{K}{R_{0}^{2}}\right)$ is Darcy's number $[18,19]$. The system of equations given by Eqns. (2) - (4) were solved subjected to Eqs. (5) - (9) in the presence of the stenosis from Eq. (1).

Assuming a constant heat flux applied on the wall, the following are the forced convection equations [21]. The equation in the inner region is given by

$$
\mathcal{E} P_{c} u_{f} \frac{\partial T_{i}}{\partial z}=\frac{1}{r} \frac{\partial}{\partial r}\left(\frac{\partial T_{i}}{\partial r}\right)
$$

and the equation in the outer region as

$$
\mathcal{E} \zeta P_{c} u_{0} \frac{\partial T_{0}}{\partial z}=\frac{1}{r} \frac{\partial}{\partial r}\left(\frac{\partial T_{0}}{\partial r}\right)
$$

where $\mathcal{E}=\frac{R_{0}}{L_{0}}, \kappa_{f}=\frac{k_{f}}{\rho_{f} c_{p}}, \zeta=\frac{\kappa_{f}}{\kappa_{0}}$ is the ratio of the diffusivities and $P_{c}=\frac{R_{0} U_{0}}{\kappa_{f}}$ is the Peclet number.

The flow rate is $Q=\pi V$, where the mean velocity, $V$, is given by

$$
V=2\left\{\int_{R_{1}}^{R} r u_{0} d r+\int_{0}^{R_{1}} r\left[(1-C) u_{f}+C u_{p}\right] d r\right\}
$$

The mean temperature, $T_{m}$, is given by

$$
T_{m}(z)=\frac{2}{V}\left\{\int_{0}^{R_{1}} u_{f} T_{i} r d r+\int_{R_{1}}^{R} u_{0} T_{0} r d r\right\}
$$

For this uniform flow, it is assumed that for all temperatures, a constant gradient (denoted by $G$ ) acts in the axial direction, that is,

$$
\frac{\partial T_{i}}{\partial z}=\frac{\partial T_{0}}{\partial z}=\frac{\partial T_{m}}{\partial z}=G
$$

The boundary conditions for the temperatures are given by

$$
\frac{\partial T_{i}}{\partial r}=0, \text { at } r=0,
$$




$$
\begin{gathered}
T_{0}=T_{i}, \text { at } r=R_{1}, \\
\varphi \frac{\partial T_{i}}{\partial r}=\frac{\partial T_{0}}{\partial r}, \text { at } r=R_{1} \\
\frac{\partial T_{0}}{\partial r}=\frac{\varphi}{\varepsilon}, \text { at } r=R, \\
T_{m}=0, \text { at } z=0 .
\end{gathered}
$$

where $\varphi=\frac{k_{f}}{k_{0}}$ is the ratio of the conductivities.

\section{Results and Discussion}

By integrating the relevant governing equations, expressions for velocities, $u_{0}, u_{f}$ and $u_{p}$ were in keeping with those of Sankar et al [20] with the mean velocity given by

$V=2\left\{\frac{k_{1}}{M}\left(R I_{1}(M R)-R_{1} I_{1}\left(M R_{1}\right)+\frac{k_{2}}{M}\left(R_{1} K_{1}\left(M R_{1}\right)-R K_{1}(M R)\right)+\frac{k_{3}}{M \gamma}\left(R_{1} J_{1}\left(M \gamma R_{1}\right)\right)-\left(\frac{d p}{d z}\right)\left(\frac{R^{2}}{2 M^{2}}+\frac{C R_{1}^{2}}{2 S}\right)\right\}\right.$

The following expressions were obtained for the temperatures,

$$
\begin{gathered}
T_{i}=G z-\frac{\varepsilon P_{c} G}{M^{2}}\left(\frac{k_{3}}{\gamma^{2}} J_{0}(M \gamma r)+\frac{r^{2} \Phi}{4}\right)+c_{1} \\
T_{0}=G z+\varepsilon \zeta P_{c} G\left(\frac{k_{1}}{M^{2}} I_{0}(M r)+\frac{k_{2}}{M^{2}} K_{0}(M r)-\frac{r^{2} \Phi}{4 M^{2}}+c_{2} \ln (r)\right)+c_{3}
\end{gathered}
$$

where $\Phi=\frac{d p}{d z}$ and $G, c_{1}, c_{2}, c_{3}, \beta_{1}, \beta_{2}, \beta_{3}, \beta_{4}, \beta_{5}, \beta_{6}$ were found by utilizing the appropriate boundary conditions. The friction-factor-Reynolds number product, $f R e$, is given by

$$
f R e=\frac{4}{\left\{\begin{array}{c}
\frac{k_{1}}{M}\left(R I_{1}(M R)-R_{1} I_{1}\left(M R_{1}\right)+\frac{k_{2}}{M}\left(R_{1} K_{1}\left(M R_{1}\right)-R K_{1}(M R)\right)\right. \\
+\frac{k_{3}}{M \gamma}\left(R_{1} J_{1}\left(M \gamma R_{1}\right)\right)-\left(\frac{d p}{d z}\right)\left(\frac{R^{2}}{2 M^{2}}+\frac{C R_{1}^{2}}{2 S}\right)
\end{array}\right\}}
$$

The average Nusselt number is used to gauge the rate of heat transfer to the blood. It was computed numerically using the formula,

$$
N u=\frac{2}{T_{0}(R, z)-T_{m}(z)}
$$

Additionally the Peclet number was assumed to be 0.03 for a microvascular flow [22]. Simpson's rule was used on the integrals which needed to be evaluated numerically. Plots for these solutions were provided using $L=1, d=0, \frac{d p}{d z}=-50, \zeta=\mathcal{E}=\varphi=1, \alpha=0.3, C=0,0.1,0.2,0.3,0.4,0.5,0.6, M=2,4,6,8$, $\delta=0,0.05,0.1,0.15,0.2, P_{c}=0.01,0.02,0.03,0.04,0.05$ and $\sqrt{D_{\mathrm{a}}}=0,0.01,0.02,0.03,0.04[8,13,18,19,21]$.

On the introduction of convective heat transfer, the following temperature profiles shown in Figs 2 and 3 were obtained. Temperature became elevated when the red blood cell count, the magnetic field strength and wall permeability increased. The trend observed in Fig. 2 is in agreement with the observations of El-Sayed et al. [12] and Sankar et al [20]. From the relationship between the two, it is clear that the thickness of the peripheral layer decreases with increasing haematocrit. Thus the temperature rises when this peripheral layer thins. 

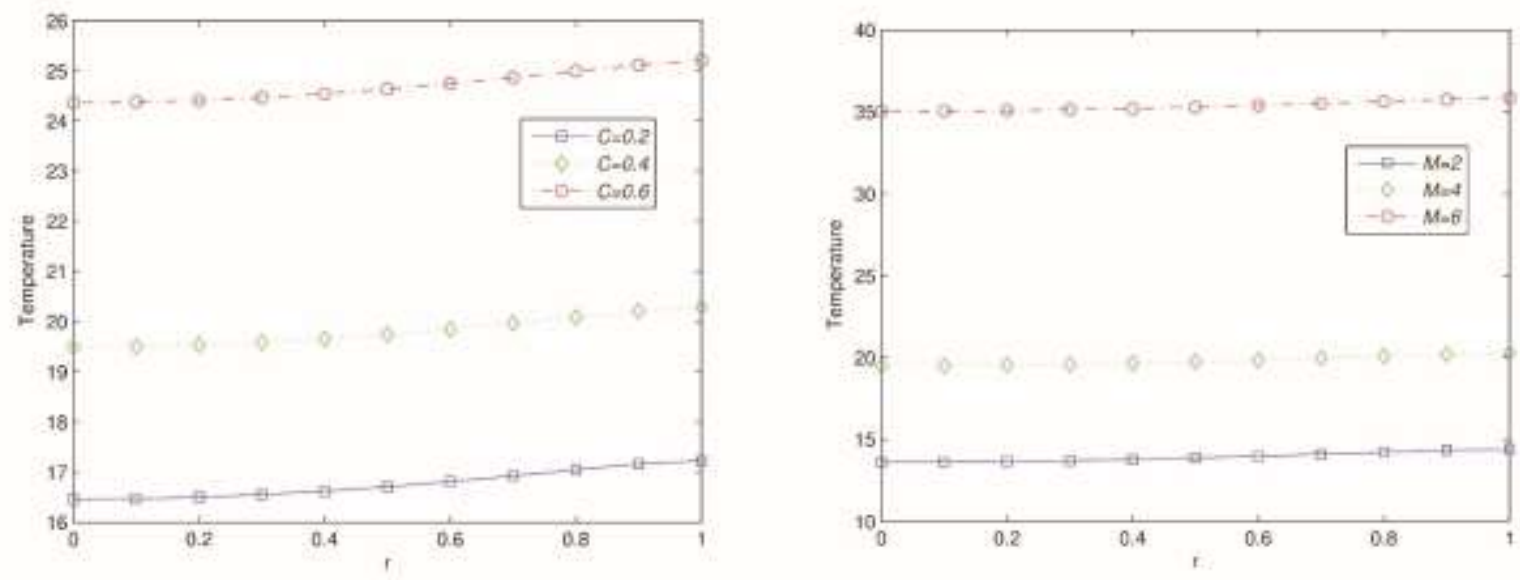

Figure 2: Variation of temperature with haematocrit (where $M=4$ and $\sqrt{D_{a}}=0.02$ ). and with Hartmann number (where $C=0.4$ and $\sqrt{D_{a}}=0.02$ ).

Figure 3 in which the temperature increases with the Darcy number indicates that temperature increases as the wall permeability increases. This must be taken into consideration and monitored in patients who take drugs which can alter wall permeability.

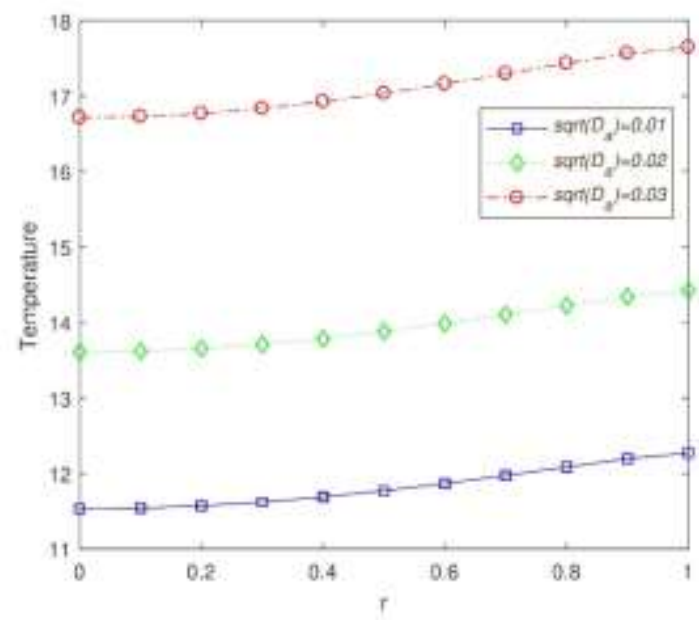

Figure 3: Variation of temperature with Darcy number (where $C=0.4$ and $M=4$ ).

In general, $f R e$ increased in the upstream of the stenosis growth and reached its maximum at the point where the stenosis height was at its greatest and then decreased downstream. It is clear from Figs 4-6 that fRe inclined with increases to haematocrit, Hartmann number, stenosis height and the Darcy number. Since $f R e$ varies inversely to the velocity, this is in keeping with previous observations of a decline in the fluid's velocity with increasing red blood cell count, magnetic field strength and stenosis growth [20]. 
Additionally, it is expected that fRe would decline as the peripheral layer thickens.
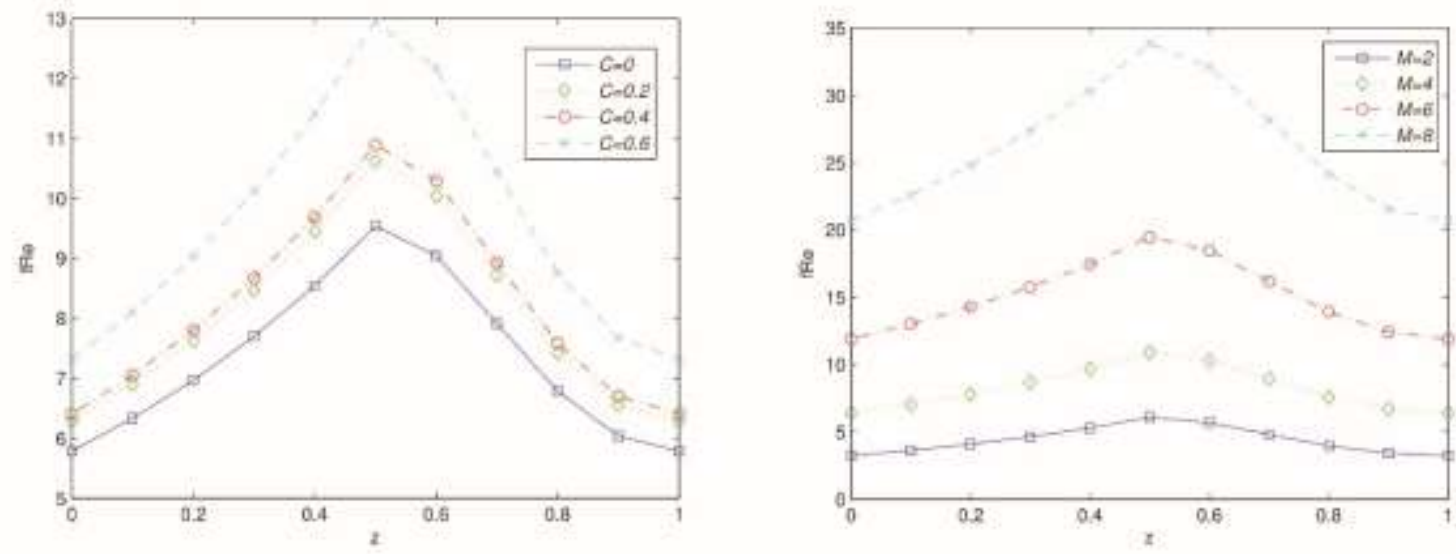

Figure 4: The effect of varying haematocrit on $f R e$ (with $\delta=0.15, M=4$ and $\sqrt{D_{a}}=0.02$ ) and varying Hartmann number on $f R e$ (with $C=0.4, \delta=0.15$ and $\sqrt{D_{a}}=0.02$ ).

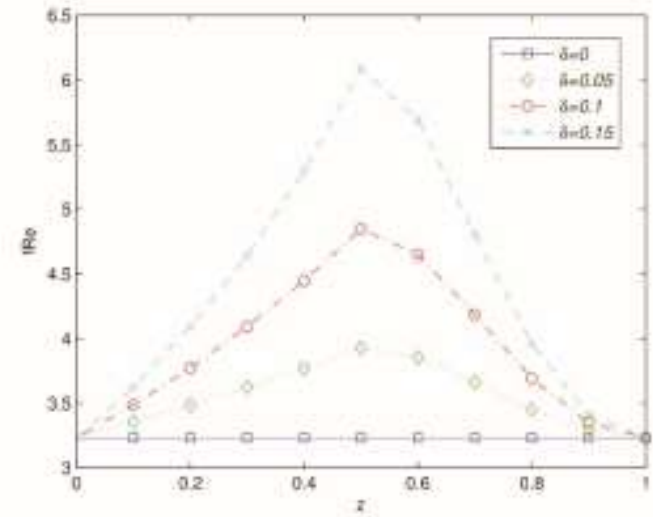

Figure 5: The effect of varying the stenosis height on $f R e$ (with $C=0.4, M=2$ and $\sqrt{D_{a}}=0.02$ )

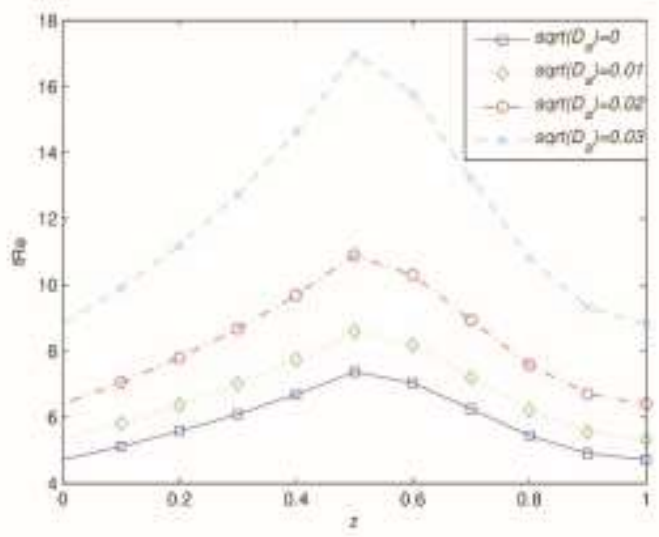

Figure 6: The effect of varying the Darcy number on $f R e$ (with $C=0.4, \delta=0.15$ and $M=2$ ).

There was a decrease in the rate of heat transfer as blood flowed through the vessel. Increasing the red blood cells in the vessel decreased the rate of heat transfer as flow progressed over the stenosis (see Fig. 7). This decrease in rate transfer was also noted by Tripathi and Kumar Sharma [17]. Thus the rate of heat transfer would increase as the peripheral layer thickens (as the red blood cell count diminishes). As the 
Hartmann number increased, the Nusselt number decreased (see Fig. 7). Thus on introducing the magnetic field and increasing its intensity, less heat and energy loss is expected.
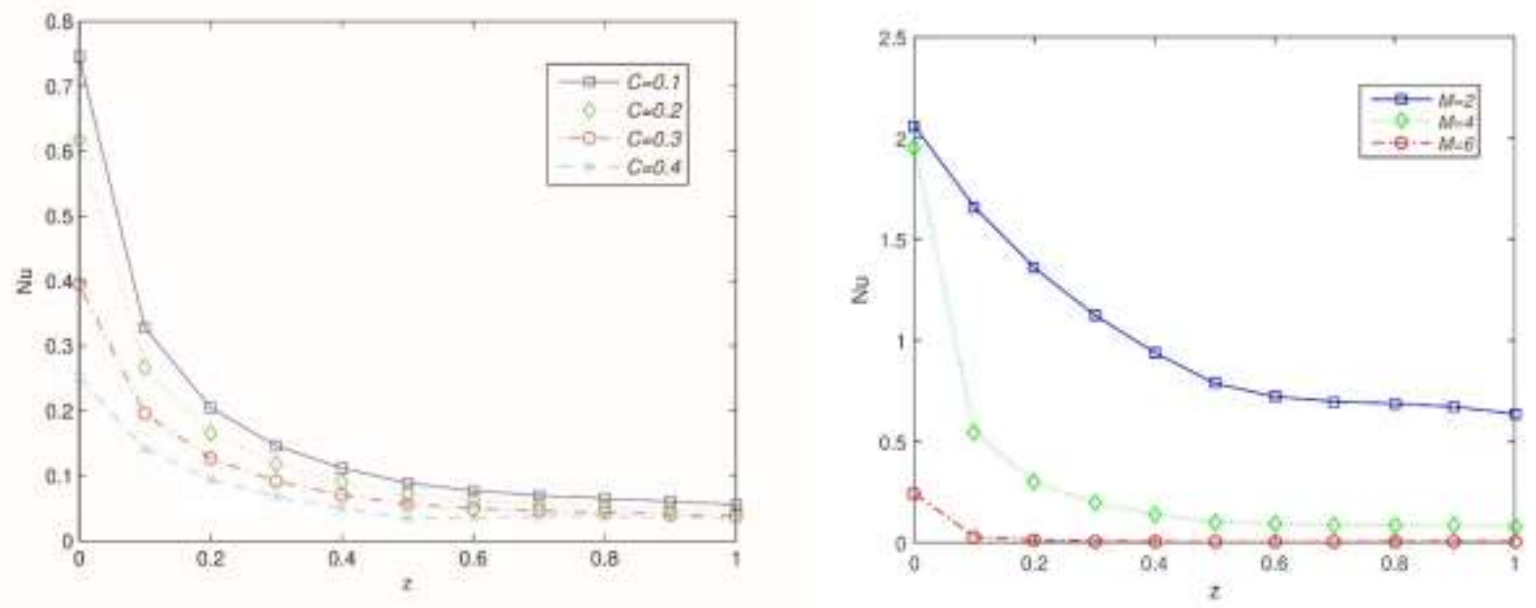

Figure 7: Variation of Nusselt number with haematocrit (where $M=8, P_{c}=0.03, \delta=0.1$ and $\sqrt{D_{a}}=0.02$ ) and with Hartmann number(where $C=0.4, P_{c}=0.03, \delta=0.1$ and $\sqrt{D_{a}}=0.03$ ).

In Fig. 8, it was observed that as the stenosis height increased there was a very small decrease in the Nusselt number. This can be useful in the analysis of heat transfer in patients with atherosclerotic plaque.

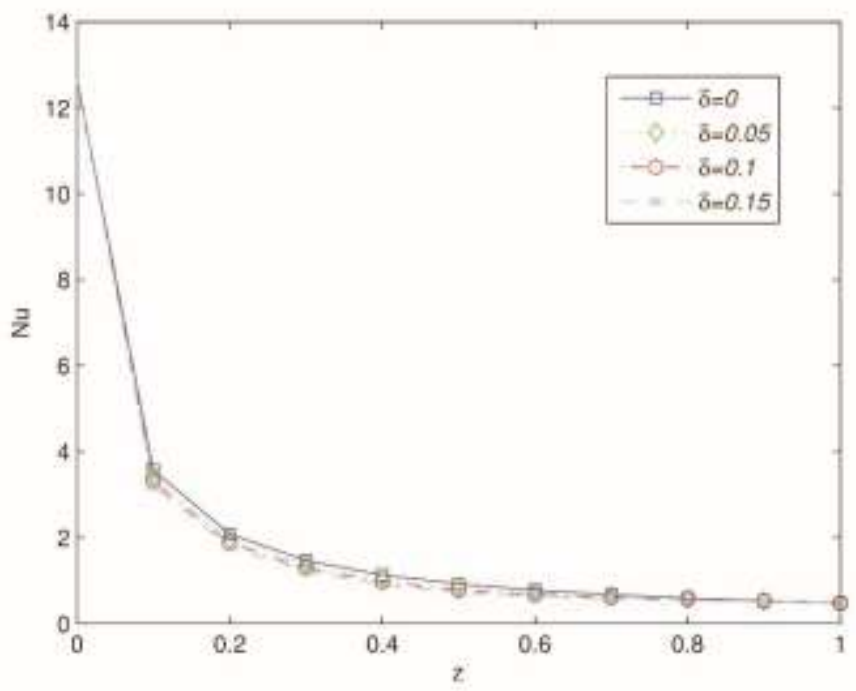

Figure 8: Variation of $N u$ with the stenosis height (where $C=0.4, P_{c}=0.03, M=4$ and $\sqrt{D_{a}}=0.02$ )

With increasing permeability reflected by increasing Darcy number in Fig. 9, the Nusselt number decreased. Thus, in the presence of an external magnetic field, the rate of heat transfer decreased as the wall became more permeable. 


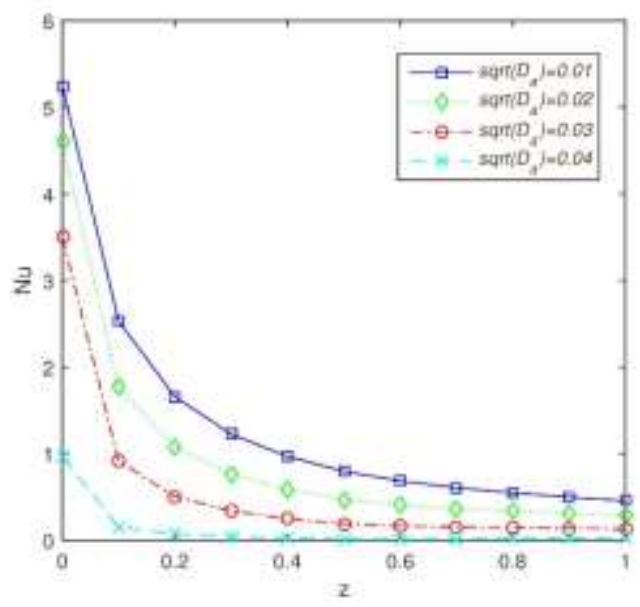

Figure 9: Variation of $N u$ with Darcy number (where $C=0.4, P_{c}=0.03, M=2$ and $\delta=0.1$ ).

\section{Conclusion}

In this study of blood flow in permeable microcirculation in the presence of a magnetic field and heat transfer where a composite stenosis was manifested, a two-layered model was used for blood. It consisted of a peripheral, Newtonian, plasma layer with a two phase, particle-fluid suspension in the core region. The fluid's temperature, friction-factor Reynolds number product and Nusselt number were examined.

The study of the effect of varying haematocrit is important to predict the effect on blood flow in patients with diseases such as polycythaemia, hypertension, plasma cell dyscrasias and Hb SS-sickle cell where haematocrit varies. In patients with an elevated red blood cell level or those exposed to high intensity magnetic fields, a rise in the temperature of the blood is possible. Increasing wall permeability increases temperature so this must be taken into consideration and monitored in patients who take drugs which can alter wall permeability. Medication such as angiotensin-converting enzyme (ACE) inhibitors, antihistimines, betablockers, calcium channel blockers, nonsteroidal anti-inflammatory drugs, decongestants and sildenafil can affect vascular permeability [23].

On introducing the magnetic field and increasing its intensity, less heat and energy loss is expected. The rate of heat transfer decreased as the wall became more permeable. As haematocrit increases or as the atherosclerotic plaque growth progresses, if the patient is exposed to heat, it can be predicted that there would be a slight decline in the heat and energy loss taking place.

The results obtained without considering the presence of a peripheral layer, stenosis, permeable walls, an external magnetic field and heat transfer can be used to analyse a mixture of particles in a fluid within a circular cylinder in any physical situation.

\section{References}

[1] J. J. W. Lagendijk. The influence of blood flow in large vessels on the temperature distribution in hyperthermia. Physics in Medicine and Biology 27, (1982) 17-23.

[2] L.M. Torell, S.K. Nilsson. Temperature gradients in low flow vessels. Physics in Medicine and Biology 23, (1987) 106-117.

[3] A. Zehforoosh, S. Hossainpour, A.A. Tahery. Numerical investigation of forced convection heat transfer for laminar flow in various parallel porous channels. International Journal of Innovation, Management and Technology 1, (2010) 252-258. 
[4] A. Raisi, B. Ghasemi, S.M. Aminossadati. A numerical study on the forced convection of laminar nanofluid in a microchannel with both slip and no-slip conditions. Numerical Heat Transfer 59, (2011) 114129.

[5] M. Yazdi, S. Abdullah, I. Hashim, K. Sopian. Effects of viscous dissipation on the slip MHD flow and heat transfer past a permeable surface with convective boundary conditions. Energies 4, (2011) 2273-2294. [6] M.M. Rahman. Locally similar solutions for hydrodynamic and thermal slip flow boundary layers over a flat plate with variable fluid properties and convective surface boundary condition. Meccanica 46, (2011) 1127-1143.

[7] M.J. Martin, I.D. Boyd. Momentum and heat transfer in a laminar boundary layer with slip flow. $J$ Thermophys and Heat Transfer 20, (2006) 710-719.

[8] R. Chaturvedi, R.K. Srivastava, V.K. Jadon. Blood flow in presence of magnetic field through porous medium and its effects on heat transfer rate. International Journal of Advanced Computer and Mathematical Sciences 3, (2012) 266-271.

[9] I.M. Eldesoky. Mathematical analysis of unsteady MHD blood flow through parallel plate channel with heat source. World Journal of Mechanics 2, (2012) 131-137.

[10] K. Das. Slip effects on MHD mixed convection stagnation point flow of a micropolar fluid towards a shrinking vertical sheet. Computers and Mathematics with Applications 63, (2012) 255-267.

[11] M. Mahmoud, S. Waheed. MHD flow and heat transfer of a micropolar fluid over a stretching surface with heat generation (absorption) and slip velocity. Journal of the Egyptian Mathematical Society 20, (2012) 20-27.

[12] M.F. El-Sayed, N. T. M. Eldabe, A.Y. Ghaly et al. Magnetothermodynamic peristaltic flow of bingham non-newtonian fluid in eccentric annuli with slip velocity and temperature jump conditions. J Mech 29, (2013) 493-506.

[13] D.N. Riahi, A.E. Garcia. 2013. Flow and heat transfer in an artery with stenosis. In 14th International Conference on Mathematics and Computers in Biology and Chemistry. MCBC.

[14] Y.S. Daniel. Steady MHD Boundary-layer slip flow and heat transfer of nanofluid over a convectively heated of a non-linear permeable sheet. Journal of Advanced Mechanical Engineering 3, (2016) 1-14.

[15] J.U. Abubakar, A.D. Adeoye. Effects of radiative heat and magnetic field on blood flow in an inclined tapered stenosed porous artery. Journal of Taibah University for Science, 14, (2020) 77-86.

[16] R. Ellahi, S.U. Rahman, S. Nadeem et al. Blood flow of nanofluid through an artery with composite stenosis and permeable walls. Applied Nanoscience 4, (2014) 919-926.

[17] B. Tripathi, B.K. Sharma. Effects of heat transfer on MHD flow of blood through an inclined porous artery with stenosis having variable viscosity. https://arxiv.org/abs/1610.03470 (accessed 11 October 2016).

[18] A. Sankar, S.R. Gunakala, D. Comissiong. Two-layered blood flow through a composite stenosis in the presence of a magnetic field. International Journal of Application or Innovation in Engineering and Management 2, (2013) 30-41.

[19] A. Sankar, S.R. Gunakala, D. Comissiong. Two-layered suspension blood flow through a composite stenosis. Journal of Mathematics Research 5, (2013) 26-38.

[20] A. Sankar, S.R. Gunakala, D. Comissiong. The effect of heat transfer on two-layered blood flow through a composite stenosis in the presence of a magnetic field. International Journal of Applied Mathematics and Statistical Sciences 3, (2014) 17-28.

[21] D.C. Sanyal, K. Das, S. Debnath. Heat transfer in a two-layered blood flow model in a narrow tube in presence of magnetic field. African Journal of Mathematical Physics 7, (2009) 1-8.

[22] M. Zakharov, and M. Sadovsky. The role of blood circulatory system in thermal regulation of animals explained by entropy production analysis, (2013) arXiv:1308.3663 [q-bio.OT].

[23] Scientific American. 2014. Common prescription drugs alter blood's flow, https://www.scientificamerican.com/article/common-prescription-drugs-alter-blood-s-flow/ 